\title{
Ultrasound-Guided Percutaneous Microwave Ablation of Bilateral Renal Cell Carcinoma: A Report of Eight Cases
}

\author{
Ruimin Zhang, ${ }^{1,2}$ Jie Yu ${ }^{3}$, Xiaoling Yu*3, Ping Liang ${ }^{3}$, Zhigang Cheng ${ }^{3}$, Zhiyu Han ${ }^{3}$, Fangyi Liu ${ }^{3}$, Shuilian \\ Tan $^{3}$ and Erpeng Q $\mathbf{i}^{1}$ \\ ${ }^{1}$ Department of Urology, Chinese People's Liberation Army General Hospital, Chinese PLA Medical School, P.R. China \\ ${ }^{2}$ Department of Urology, Baotou Fourth Hospital, P.R. China
}

${ }^{3}$ Department of Interventional Ultrasound, Chinese People's Liberation Army General Hospital, P.R. China

*Corresponding author: Xiaoling Yu, Department of Interventional Ultrasound, Chinese People's Liberation Army General Hospital, NO.28 Fuxing Road, Haidian District, Beijing 100853, P.R. China

\begin{abstract}
ARTICLE INFO
Received: 杫 November 03, 2019

Published: 㹃 November 12, 2019

Citation: Ruimin Zhang, Jie Yu, Xiaoling Yu, Ping Liang, Zhigang Cheng, Zhiyu Han, et al. Ultrasound-Guided Percutaneous Microwave Ablation of Bilateral Renal Cell Carcinoma: A Report of Eight Cases. Biomed J Sci \& Tech Res 22(5)-2019. BJSTR. MS.ID.003802.
\end{abstract}

Keywords: Microwaves; Ultrasonography; Kidney; Renal Cell Carcinoma

Abbreviations: RCC: Renal Cell Carcinomas; MWA: Microwave Ablation; RFA: Radiofrequency Ablation; US: Ultrasound; CT: Computed Tomography; MRI: Magnetic Resonance Imaging

\section{ABSTRACT}

Objective: We reviewed a series of 8 cases of bilateral renal cell carcinomas (RCCs) to evaluate the safety and outcomes of ultrasound-guided percutaneous microwave ablation (MWA) techniques, which is an important alternative to tumor control in localities and renal function preservation interventions.

Materials and Methods: Eight patients of bilateral RCCs treated with percutaneous microwave ablation under ultrasound guidance in our department between November 2012 and October 2018 were reviewed retrospectively. The tumor diameters range from 0.7 to $6.2 \mathrm{~cm}$. Microwaves were emitted at 45-60W for 300 seconds and prolonged as necessary to attain temperatures sufficient to ensure tumor kill. Renal functions including serum creatinine and urea levels before MWA and one day after MWA were collected. The treatment efficacy and technical success were evaluated by contrast enhanced ultrasound and computed tomography or magnetic resonance imaging.

Results: All tumors were completely ablated 2MWA sessions; 2 recurrences were observed. No severe complications occurred during MWA procedure. No significant differences in the post-MWA renal functions compared with the pre-MWA levels. The ablation zone was gradually shrank with time.

Conclusion: Ultrasound guided percutaneous MWA appears to be a safe and effective technique for bilateral renal cell carcinomas. Personalized MWA can achieve good local tumor control, renal function preservation and low complication rate.

\section{Introduction}

Bilateral renal cell carcinomas (RCCs) are clinically uncommon, accounting for $1 \%-5 \%$ of renal cell carcinomas [1,2]. Compared to unilateral RCC, bilateral RCCs presents a surgical challenge in balancing the need for long-term tumor control and preserving maximal renal functions. The optimal management of bilateral RCC remains controversial. Given the surgical constraints, nephron-sparing surgery and image-guided thermal ablation have increasing popularity in recent years due to its safety and efficacy for bilateral RCC, patients having elder age or with comorbidities [3-7]. Compared with nephron-sparing surgery, image-guided thermal ablation, such as percutaneous microwave ablation (MWA), radiofrequency ablation (RFA), and cryoablation, has showed similar good effects on local tumor control, but less invasive and low incidence of major complications in the treatment of RCC [8- 
10]. MWA ablation technique directly destroy a large lesion near the antenna in a short time, and MWA energy propagation is rarely limited by tissue desiccation and charring [11]. MWA ablation techniques and devices have undergone significant improvements. And ultrasound (US) guided percutaneous MWA is now a wellestablished technique for treating small renal tumors $[10,12]$. To the best of our knowledge, there have been no published reports concerning US guided percutaneous MWA ablation in the treatment of bilateral RCCs. In this study, we aimed to evaluate the safety and clinical outcomes of patients with bilateral RCCs with US guided percutaneous MWA ablation.

\section{Patients and Methods}

\section{Subjects}

All cases of bilateral RCCs treated with US guided percutaneous MWA at the Department of Urology at the General Hospital of the People's Liberation Army during period of November 2012 and October 2018 were reviewed. The retrieval of data was in concordance with institutional IRB regulations. All tumors were histopathologically proven by 18-gauge core needle biopsies under US guidance before the ablation. Renal functions including serum creatinine and urea levels before MWA and one day after MWA were retrospectively retrieved from case records.

\section{Imaging Examination}

Before treatment, all patients were subjected to chest x-ray, abdominal ultrasonography, computed tomography (CT), and/or magnetic resonance imaging (MRI) were performed to evaluate bilateral kidneys and the existence of metastasis.

\section{MWA Ablation Protocol}

The MWA system (KY-2000; Nanjing Kangyou Medical Treatment Equipment Co., Ltd., Nanjing, China), consisting of MWA generators, flexible coaxial cables and water-pumping machines, was used to produce the highest power output of $100 \mathrm{~W}$ at $2450 \mathrm{MHz}$. The 15-gauge diameter cooled-shaft needle antenna $(18 \mathrm{~cm}$ length, $11 \mathrm{~mm}$ active tip) was applied in this study. Lesions for MWA ablation were decided according to the diagnoses of the tumor on contrast-enhanced imaging pre-MWA. In general, for tumors less than $2.0 \mathrm{~cm}$ in diameter, a single antenna was advanced, whereas two or more antennas were required for tumors with diameters of $2.0 \mathrm{~cm}$ or greater. All patients were kept under intravenous anaesthesia and a combination of Propofol (Diprivan; Zeneca Pharmaceuticals, Wilmington, DE) and Ketamine (Shuanghe Pharmaceuticals, Beijing, China) was administered by an anaesthesiologist. The antenna was percutaneously advanced into the index tumor under US guidance. MWA output was emitted at a power of 45-60W for 300 seconds and prolonged as necessary to attain temperatures sufficient to ensure tumor kill. When the hyperechoic zone covered the entire index tumor on US imaging, the ablation was ceased. Then a needle tract cauterization was routinely performed during the antenna withdrawal to minimize bleeding and tumor seeding along the needle track.

\section{Follow-up}

US imaging, contrast-enhanced CT or MRI was routinely employed on day1-day3 after the MWA ablation procedure to evaluate the treatment efficacy and technical success. An irregular peripheral enhancement of the ablated lesion indicated a possible residual unablated tumor; consequently, an additional MWA session was repeated within 1 week.

\section{Statistical Analysis}

The measurement data were presented as the mean \pm SD and compared with Student's t-test or Mann-Whitney test where appropriate. Statistical analysis was performed using SPSS $11.0 \mathrm{soft}$ ware for windows (SPSS Inc., Chicago, IL, USA). A p value less than 0.05 was considered statistically significant.

\section{Results}

\section{Patient Demographics}

In the present study, 8 patients $(7$ males and 1 female; mean age $59.4 \pm 9.9$ years) who had bilateral RCCs were included for analysis (Table 1). Three patients (1 patient had received unilateral nephrectomy and 2 patients had received bilateral nephrectomy) had 1 tumor. Two patients had 2 tumors. Three patients had four tumors. The tumor diameters range from 0.7 to $6.2 \mathrm{~cm}$. Amongst them, the hisological subtype was clear cell carcinoma (19/19).

Table 1: Clinical features of the patients and tumors.

\begin{tabular}{|c|c|}
\hline Variables & Value \\
\hline Gender (male/female) & $7 / 1$ \\
\hline Mean age \pm SD (range, years) & $59.4 \pm 9.9(30-81)$ \\
\hline Total no. of tumors & 19 \\
\hline Mean maximum tumor diameter \pm SD (range, cm) & $2.84 \pm 1.54(0.7-6.2)$ \\
\hline Ablated tumor with one MWA session (\%) & $17(89.5 \%)$ \\
\hline Ablated tumors with one MWA session (\%) & $2(10.5 \%)$ \\
\hline Mean follow-up \pm SD (range) (months) & $27.3 \pm 14.6(7-59)$ \\
\hline
\end{tabular}

\section{Outcomes of MWA Ablation}

In total, 19 lesions (10 lesions were in the right kidney, and 9 lesions were in the left kidney) were ablated using 21 US guided percutaneous MWA sessions (Table 1). One session was performed to ablate $89.5 \%$ of the RCCs (17/19), and an additional session was repeated for the incompletely destroyed tumors because a residual ablated tumor was detected by US imaging within 3 days after the MWA. All lesions necrosed after MWA ablation (Figure 1). After MWA, there were 5 patients with mild to moderate fever within $24 \mathrm{~h}$ and 1 patient with severe fever in for 9 days. No severe complications occurred during and after MWA procedure. The median follow-up time was 24 months (ranged from 7 months to 59 months). During the follow-up, there was no local tumor 
recurrence in 6 of 8 completely ablated tumor lesions. One patient was retreated by MWA 6 months after ablation, and another was retreated by MWA 19 months after ablation due to local tumor recurrence (Figure 2). No patients lost their life after MWA.

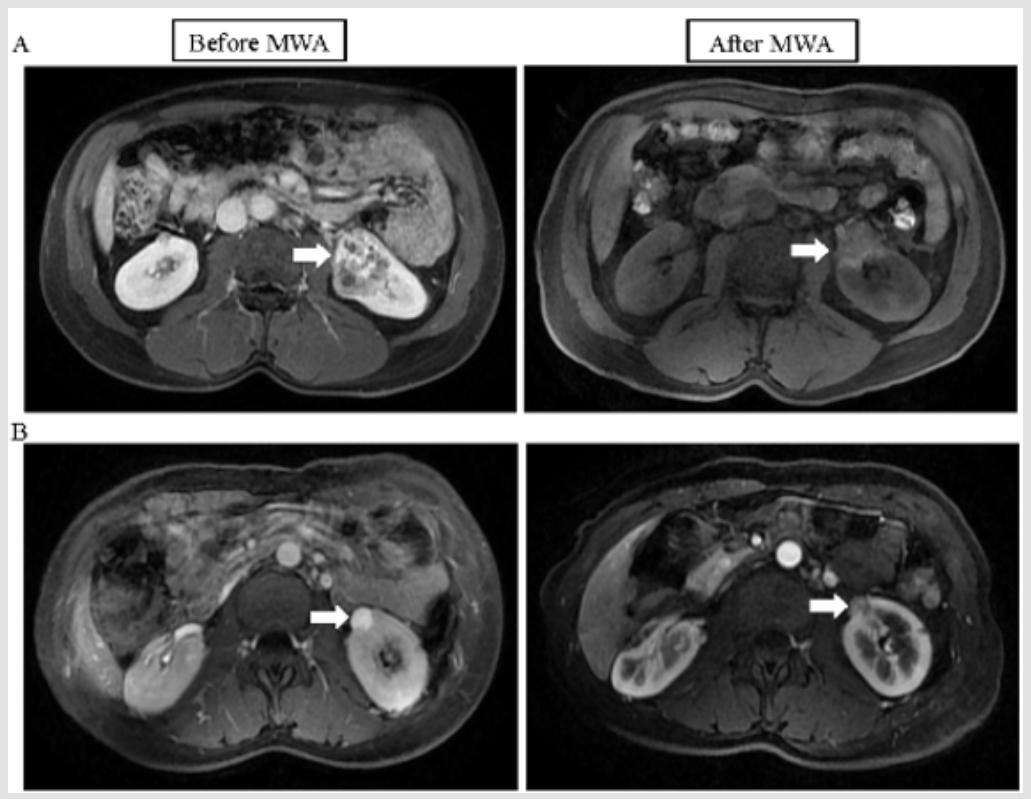

Figure 1: Pre-MWA and post-MWA treatment MRI images of two patients with RCCs. (A) Pre-MWA MRI image showing a tumor (white arrow), with a maximal diameter of $58 \mathrm{~mm}$, in the left kidney of a 44-year-old male (left). Post-MWA MRI image showing ablated lesion hypointensity (white arrow) at 1 month (right). (B) Pre-MWA MRI image showing a tumor (white arrow), with a maximal diameter of $40 \mathrm{~mm}$, in the left kidney of a 34-year-old female (left). Post-MWA MRI image showing ablated lesion hypointensity in the ablation zone (white arrow) on day 3 (right). MRI, magnetic resonance imaging; MWA, percutaneous microwave ablation; RCC, renal cell carcinomas.
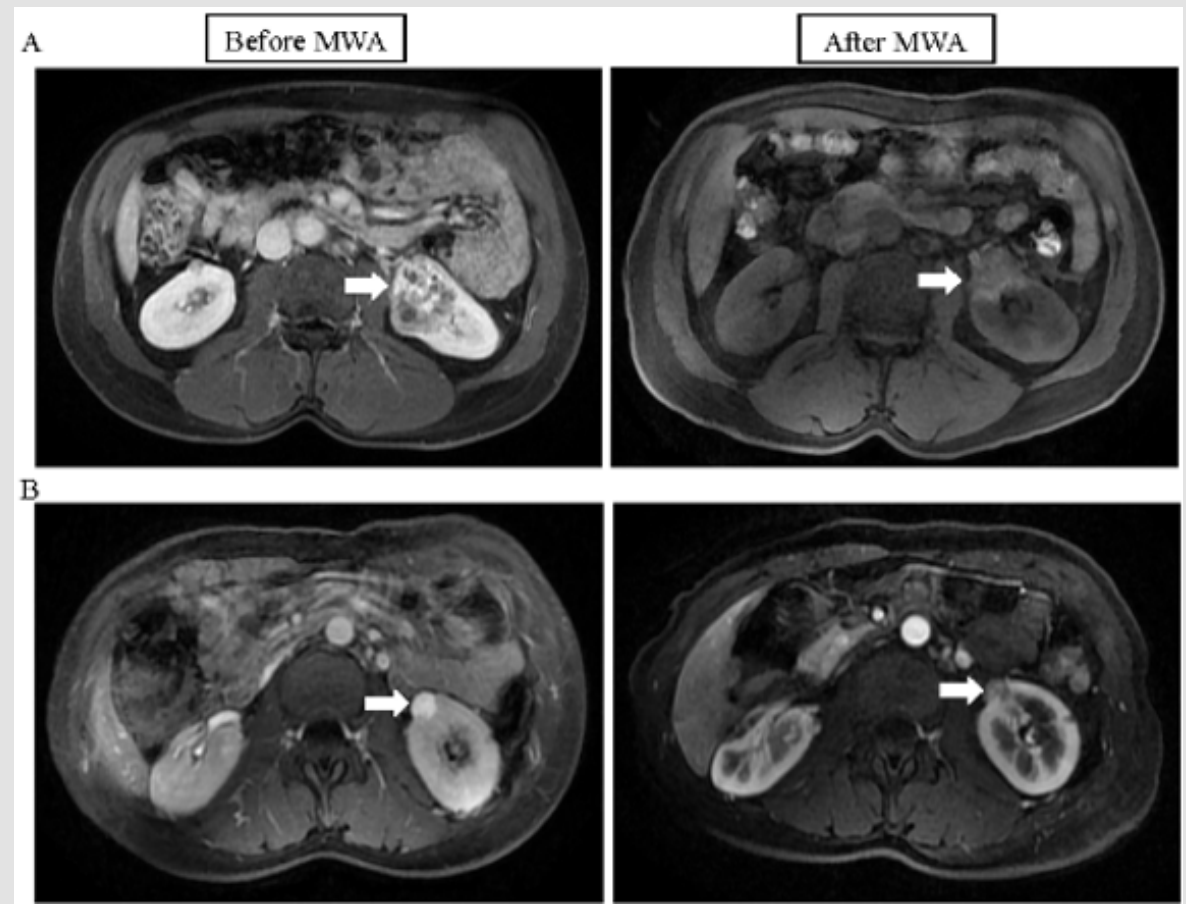

Figure 2: MRI images of a RCC with a maximal diameter of $84 \mathrm{~mm}$, prior to, following and retreated by MWA. (A) Pre-MWA MRI image showing a tumor (white arrow) in the left kidney of a 48-year-old male (left). Post-MWA MRI image showing necrotic lesions (white arrow) on day 3 (right). (B) At 6 months after MWA, MRI image showing a local tumor recurrence with a maximal diameter of $27 \mathrm{~mm}$ in the right kidney (left). After MWA retreatment, MRI image showing no residual tumor in the ablation zone (white arrow) on day 3 (right). MRI, magnetic resonance imaging; MWA, percutaneous microwave ablation; $\mathrm{RCC}$, renal cell carcinomas. 


\section{Renal Function}

After MWA treatment, no obvious changes of renal function were observed in all patients. Compared with the mean levels prior to the
MWA, there were no significant differences in the mean serum $\mathrm{Cr}$ and BUN levels 1 day after the MWA procedure $(6.48 \pm 1.93 \mathrm{lmol} / \mathrm{L}$ versus $6.17 \pm 0.82 \mathrm{lmol} / \mathrm{L}, \mathrm{p}=0.683 ; 107.4 \pm 27.90 \mathrm{mmol} / \mathrm{L}$ versus $124.43 \pm 39.51 \mathrm{mmol} / \mathrm{L}, \mathrm{p}=0.335$ ) (Figure 3 ).
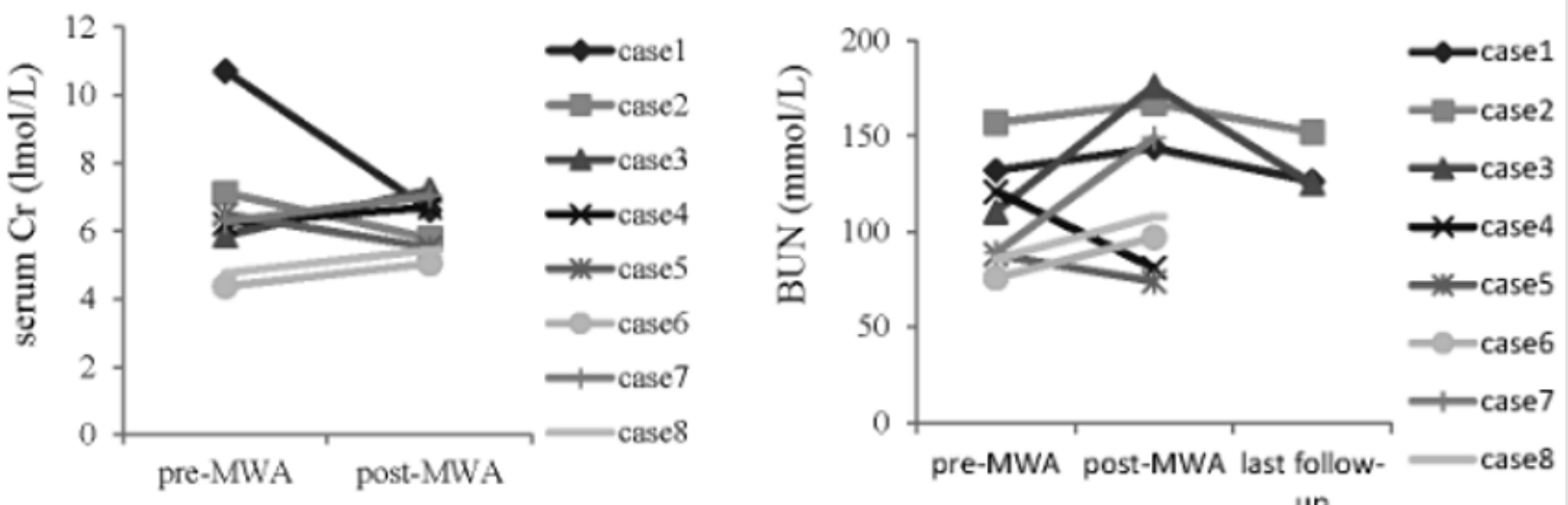

Figure 3: The levels of serum Cr and BUN in 8 patients with bilateral RCCs pre-MWA and post-MWA treatment. Cr, creatinine; BUN, blood urea nitrogen.

\section{Discussion}

For the majority of patients with bilateral RCCs, partial or complete nephrectomy has been considered the first choice of treatment $[13,14]$. However, certain patients are non-surgical candidates or refuse surgery due to comorbidities and cosmetic requirements. As non-surgical treatments, image-guided ablative treatments, including MWA, radiofrequency ablation and cryrotherapy, have been advocated as minimally invasive. While ultrasound-guided percutaneous MWA techniques have gained popularity as treatment for renal cell carcinomas in recent years due to its safety and effectiveness, there is limited practices in performing such techniques in bilateral RCCs. In this context, US guided percutaneous MWA was used to treat bilateral RCCs. In this study, up to $75 \%(6 / 8)$ of patients treated with MWA have good outcomes upon long-term follow up. Two patients had more than 1 year follow-up with no tumor regrowth after received the second or the third MWA because of the tumor recurrence. One must appreciate that MWA for bilateral RCCs is technically demanding, and thus $75 \%$ of patients had good recovery is encouraging. Other advantages of microsurgery include renal function preservation and no severe complications. US-guided MWA has been applied to assist tumor ablation of liver, kindey, and benign prostatic hyperplasia [15-17]. Cheng also reported 24 cases of RCCs adjacent to the intestinal tract using US-guided MWA treatment [7]. In this series, the use of percutaneous under the guidance of US, complete ablation was successfully achieved in the tumor lesions and no tumor recurrence was detected during the follow-up.

The percutaneous US-guided MWA did not affect the residual renal function and pose injury to the adjacent organ [7]. In the present study, seven tumor lesions close to intestinal tract and 2 lesions close to liver. US imaging had helped to identify these cases and these tumors were completely ablated without posing injury to the adjacent organ. We analyzed the change of renal function preMWA and post-MWA of each patient, no significant elevation was observed. The level of three patients' serum creatinine increased lightly, but decreased to level of pre-MWA in the last follow-up. Hence, clinicians alike should solicit the support from US-guided MWA for patients with complex renal tumors or renal dysfunction were attempted, and this would identify anatomy and improve overall surgical outcomes. Despite these successes, one must also reckon the small population size of bilateral RCCs reported in this series, further intensive study and well-designed prospective study should be performed to identify the effect of US-guided percutaneous MWA in the future.

\section{Conclusion}

Based on these favorable follow-up outcomes, the thermal technique would not harm the residual function and pose unacceptable damage to the adjacent organs. The treatment procedure could be individually tailored, according to the location and size of the tumor. US-guided percutaneous MWA is effective and safe to treat the patients with bilateral RCCs or the patients who lost the chance of surgery can have a positive outcome with correctly selected techniques.

\section{Acknowledgment}

The authors thank the Interventional Ultrasound, Diagnostic Radiology Department and the Nuclear Medicine Department of the General Hospital of the People's Liberation Army for their assistance. 


\section{Declaration of Interest}

The authors report no other declarations of interest. The authors alone are responsible for the content and writing of the paper.

\section{Compliance with Ethical Standards}

The use of human clinical materials in this study was approved by the Ethical Committee of the General Hospital of the People's Liberation Army. All patients or their caregivers have provided written informed consent.

\section{References}

1. Klatte T, Wunderlich H, Patard JJ, Kleid MD, Lam JS, et al. (2007) Clinicopathological features and prognosis of synchronous bilateral renal cell carcinoma: an international multicentre experience. BJU Int 100(1): 21-25.

2. Lowrance WT, Yee DS, Maschino AC, Cronin AM, Bernstein M, et al. (2010) Developments in the surgical management of sporadic synchronous bilateral renal tumors. BJU Int 105(8): 1093-1097.

3. Boorjian SA, Crispen PL, Lohse CM, Leibovich BC, Blute ML (2008) The impact of temporal presentation on clinical and pathological outcomes for patients with sporadic bilateral renal masses. Eur Urol 54(4): 855863.

4. Wang B, Gong H, Zhang X, Li H, Ma X, et al. (2016) Bilateral synchronous sporadic renal cell carcinoma: retroperitoneoscopic strategies and intermediate outcomes of 60 patients. PLoS One 11(5): e0154578.

5. Vetterlein MW, Jindal T, Becker A, Regier M, Kluth LA, et al. (2016) Small renal masses in the elderly: contemporary treatment approaches and comparative oncological outcomes of nonsurgical and surgical strategies. Investig Clin Urol 57(4): 231-239.

6. D'Andrea D, Shariat SF, Klatte T (2016) Update on ablative therapies of renal tumors. Curr Opin Urol 26(5): 410-416.

7. Cheng Z, Yu X, Han Z, Liu F, Yu J, et al. (2017) Ultrasound-guided hydro dissection for assisting percutaneous microwave ablation of renal cell

ISSN: 2574-1241

DOI: $10.26717 /$ BJSTR.2019.22.003802

Xiaoling Yu. Biomed J Sci \& Tech Res

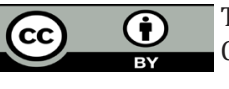

This work is licensed under Creative

Commons Attribution 4.0 License

Submission Link: https://biomedres.us/submit-manuscript.php carcinomas adjacent to intestinal tracts: a preliminary clinical study. Int J Hyperthermia 34(3):315-320.

8. Georgiades C, Rodriguez R (2013) Renal tumor ablation. Tech Vasc Interv Radiol 16(4): 230-238.

9. Wah TM, Irving HC, Gregory W, Cartledge J, Joyce AD, et al. (2014) Radiofrequency ablation (RFA) of renal cell carcinoma (RCC): experience in 200 tumours. BJU Int 113(3): 416-428.

10. Lin Y, Liang P, Yu XL, Yu J, Cheng ZG, et al. (2014) Percutaneous microwave ablation of renal cell carcinoma is safe in patients with a solitary kidney. Urology 83(2): 357-363.

11. Liu FY, Yu XL, Liang P, Wang Y, Zhou P et al. (2010) Comparison of percutaneous $915 \mathrm{MHz}$ microwave ablation and $2450 \mathrm{MHz}$ microwave ablation in large hepatocellular carcinoma. Int J Hyperthermia 26(5): 448455 .

12. Dong X, Li X, Yu J, Ming-an Yu, Xiaoling Yu, et al. (2016) Complications of ultrasound guided percutaneous microwave ablation of renal cell carcinoma. Onco Targets Ther 9: 5903-5909.

13. Qi N, Li T, Ning X, Peng X, Cai L, et al. (2017) Clinicopathologic Features and Prognosis of Sporadic Bilateral Renal Cell Carcinoma: A Series of 148 Cases. Clin Genitourin Cancer 15(5) :618-624.

14. Babaian KN, Merrill MM, Matin S, Pheroze Tamboli, Nizar M Tannir, et al. (2014) Partial nephrectomy in the setting of metastatic renal cell carcinoma. J Urol 192(1): 36-42.

15. Cheng Z, Liang P, Yu X, Han Z, Liu F, et al. (2017) Percutaneous microwave ablation for benign focal liver lesions: Initial clinical results. Oncol Lett 13(1): 429-434.

16. Chen CN, Liang P, Yu J, Yu XL, Cheng ZG, et al. (2016) Contrast-enhanced ultrasound-guided percutaneous microwave ablation of renal cell carcinoma that is inconspicuous on conventional ultrasound. Int J Hyperthermia 32: 607-613.

17. Garnon J, Koch G, Caudrelier J, Ramamurthy N, Rao P, et al. (2016) Percutaneous imageguided cryoablation of challenging mediastinal lesions using large volume hydrodissection: technical considerations and outcomes. Cardiovasc Intervent Radiol 39(11): 1636-1643.

$\begin{array}{ll}\text { BIOMEDICAL } & \text { Assets of Publishing with us } \\ \text { RESEARCHES } & \text { - Global archiving of articles } \\ & \text { - Immediate, unrestricted online access } \\ & \text { - Rigorous Peer Review Process } \\ & \text { - Authors Retain Copyrights } \\ \end{array}$

